University of Wollongong

Research Online

Faculty of Health and Behavioural Sciences -

Papers (Archive)

Faculty of Science, Medicine and Health

$1-1-2012$

\title{
Study protocol for a randomized controlled trial of humor therapy in residential care: the Sydney Multisite Intervention of LaughterBosses and ElderClowns (SMILE)
}

\author{
Belinda Goodenough \\ University Of New South Wales \\ Lee-Fay Low \\ University Of New South Wales \\ Anne-Nicole Casey \\ University Of New South Wales \\ Lynn Chenoweth \\ University Of Technology Sydney, Lynette.Chenoweth@uts.edu.au \\ Richard Fleming \\ University of Wollongong, rfleming@uow.edu.au
}

See next page for additional authors

Follow this and additional works at: https://ro.uow.edu.au/hbspapers

Part of the Arts and Humanities Commons, Life Sciences Commons, Medicine and Health Sciences Commons, and the Social and Behavioral Sciences Commons

\section{Recommended Citation}

Goodenough, Belinda; Low, Lee-Fay; Casey, Anne-Nicole; Chenoweth, Lynn; Fleming, Richard; Spitzer, Peter; Bell, Jean-Paul; and Brodaty, Henry: Study protocol for a randomized controlled trial of humor therapy in residential care: the Sydney Multisite Intervention of LaughterBosses and ElderClowns (SMILE) 2012, 2037-2044.

https://ro.uow.edu.au/hbspapers/3162

Research Online is the open access institutional repository for the University of Wollongong. For further information contact the UOW Library: research-pubs@uow.edu.au 


\title{
Study protocol for a randomized controlled trial of humor therapy in residential care: the Sydney Multisite Intervention of LaughterBosses and ElderClowns (SMILE)
}

\begin{abstract}
Background: Humor therapy is a non-pharmacological intervention with potential to improve mood and quality of life for institutionalized older persons, including those with dementia. The primary aims of the Sydney Multisite Intervention of LaughterBosses and ElderClowns (SMILE) are to examine the effects of humor therapy on residents' mood, quality of life, social engagement, and agitation. Methods: SMILE is a single-blinded cluster-randomized controlled trial where 398 consented residents in 35 residential aged care facilities will be allocated to receive humor therapy or usual care. Residents allocated to the intervention group will engage in humor therapy with professional performers (ElderClowns) and trained facility staff (LaughterBosses) for a minimum of nine two-hour sessions over 12 weeks as well as engaging humorously with LaughterBosses during the course of daily care. The usual care control group will not engage in any formal humor therapy. Researchers, blind to treatment allocation, will assess residents at baseline (week 0), post-intervention (week 13), and follow-up (week 26). The measurement suite includes the Cornell Scale for Depression in Dementia, the Dementia Quality of Life Scale, the Multidimensional Observation Scale for Elderly Subjects, the Cohen-Mansfield Agitation Inventory, and the Neuropsychiatric Inventory. Observations of residents' engagement will be recorded at each humor therapy session. Conclusions: SMILE is the first large rigorous study of humor therapy in aged care. (C) International Psychogeriatric Association 2012.
\end{abstract}

\section{Keywords}

trial, controlled, randomized, laughterbosses, protocol, smile, intervention, elderclowns, multisite, study, sydney, care, residential, therapy, humor

\section{Disciplines}

Arts and Humanities | Life Sciences | Medicine and Health Sciences | Social and Behavioral Sciences

\section{Publication Details}

Goodenough, B., Low, L., Casey, A., Chenoweth, L., Fleming, R., Spitzer, P., Bell, J. \& Brodaty, H. (2012). Study protocol for a randomized controlled trial of humor therapy in residential care: the Sydney Multisite Intervention of LaughterBosses and ElderClowns (SMILE). International Psychogeriatrics, 24 (12), 2037-2044.

\section{Authors}

Belinda Goodenough, Lee-Fay Low, Anne-Nicole Casey, Lynn Chenoweth, Richard Fleming, Peter Spitzer, Jean-Paul Bell, and Henry Brodaty 


\section{International Psychogeriatrics}

http://journals.cambridge.org/IPG

Additional services for International Psychogeriatrics:

Email alerts: Click here

Subscriptions: $\underline{\text { Click here }}$

Commercial reprints: $\underline{\text { Click here }}$

Terms of use : $\underline{\text { Click here }}$

\section{Study protocol for a randomized controlled trial of humor therapy in residential care: the Sydney Multisite Intervention of LaughterBosses and ElderClowns (SMILE)}

Belinda Goodenough, Lee-Fay Low, Anne-Nicole Casey, Lynn Chenoweth, Richard Fleming, Peter Spitzer, Jean-Paul Bell and Henry Brodaty

International Psychogeriatrics / Volume 24 / Issue 12 / December 2012, pp 2037 - 2044

DOI: 10.1017/S1041610212000683, Published online:

Link to this article: http://journals.cambridge.org/abstract_S1041610212000683

How to cite this article:

Belinda Goodenough, Lee-Fay Low, Anne-Nicole Casey, Lynn Chenoweth, Richard Fleming, Peter Spitzer, Jean-Paul Bell and Henry Brodaty (2012). Study protocol for a randomized controlled trial of humor therapy in residential care: the Sydney Multisite Intervention of LaughterBosses and ElderClowns (SMILE). International Psychogeriatrics, 24, pp 2037-2044 doi:10.1017/S1041610212000683

Request Permissions : $\underline{\text { Click here }}$ 


\title{
PROTOCOL-ONLY PAPER
}

\section{Study protocol for a randomized controlled trial of humor therapy in residential care: the Sydney Multisite Intervention of LaughterBosses and ElderClowns (SMILE)}

\author{
Belinda Goodenough, ${ }^{1}$ Lee-Fay Low, ${ }^{1}$ Anne-Nicole Casey, ${ }^{1}$ Lynn Chenoweth, ${ }^{2,3}$ \\ Richard Fleming, ${ }^{4}$ Peter Spitzer, ${ }^{5}$ Jean-Paul Bell ${ }^{6}$ and Henry Brodaty ${ }^{1,7}$ \\ ${ }^{1}$ Dementia Collaborative Research Centre, School of Psychiatry, University of New South Wales, Sydney, New South Wales, Australia \\ ${ }^{2}$ Faculty of Nursing, Midwifery and Health, University of Technology Sydney, Sydney, New South Wales, Australia \\ ${ }^{3}$ South Eastern Sydney Local Health District, Sydney, New South Wales, Australia \\ ${ }^{4}$ NSW/ACT Dementia Training Study Centre, University of Wollongong, Wollongong, New South Wales, Australia \\ ${ }^{5}$ The Humour Foundation, Chatswood, New South Wales, Australia \\ ${ }^{6}$ Arts Health Institute, Newcastle, New South Wales, Australia \\ ${ }^{7}$ Academic Department for Old Age Psychiatry, Prince of Wales Hospital, Randwick, New South Wales, Australia
}

ABSTRACT

Background: Humor therapy is a non-pharmacological intervention with potential to improve mood and quality of life for institutionalized older persons, including those with dementia. The primary aims of the Sydney Multisite Intervention of LaughterBosses and ElderClowns (SMILE) are to examine the effects of humor therapy on residents' mood, quality of life, social engagement, and agitation.

Methods: SMILE is a single-blinded cluster-randomized controlled trial where 398 consented residents in 35 residential aged care facilities will be allocated to receive humor therapy or usual care. Residents allocated to the intervention group will engage in humor therapy with professional performers (ElderClowns) and trained facility staff (LaughterBosses) for a minimum of nine two-hour sessions over 12 weeks as well as engaging humorously with LaughterBosses during the course of daily care. The usual care control group will not engage in any formal humor therapy. Researchers, blind to treatment allocation, will assess residents at baseline (week 0), post-intervention (week 13), and follow-up (week 26). The measurement suite includes the Cornell Scale for Depression in Dementia, the Dementia Quality of Life Scale, the Multidimensional Observation Scale for Elderly Subjects, the Cohen-Mansfield Agitation Inventory, and the Neuropsychiatric Inventory. Observations of residents' engagement will be recorded at each humor therapy session.

Conclusions: SMILE is the first large rigorous study of humor therapy in aged care.

Key words: aged care, nursing home, dementia, behavioral and psychological symptoms of dementia (BPSD), psychosocial interventions

\section{Introduction}

The rising prevalence of dementia is a major challenge, creating an unprecedented demand for dementia care in residential aged care facilities (RACFs). Residential care for older persons is common. At age 65, women have a 50\% risk and men have a $32 \%$ risk of entering an RACF in their remaining lifetime (Cullen, 2007). Once admitted, women stay in a facility for three and a half years

Correspondence should be addressed to: Dr. Lee-Fay Low, Dementia Collaborative Research Centre, School of Psychiatry, University of New South Wales, Sydney, New South Wales 2052, Australia. Phone: +61 2 93852599; Fax: + 612 93852200. Email: Lf.Low@unsw.edu.au. Received 23 Nov 2011; revision requested 19 Feb 2012; revised version received 20 Mar 2012; accepted 21 Mar 2012. First published online 14 May 2012. and men for two years and four months on average (Cullen, 2007).

Increasing demand and costs of dementia care services are forcing policy-makers and service providers to look for research guidance regarding the best types of dementia care that are cost effective and sustainable, and that also promote optimal quality of life for people with dementia. The residential aged care sector struggles to maintain quality of life for residents while operating within fiscal restraints, staffing challenges, and increasing consumer demands (Braithwaite, 2001).

More than $40 \%$ of aged care residents have been reported to experience depression (Snowdon and Fleming, 2008), and around 90\% manifest one or more behavioral or psychological symptom 
of dementia (Brodaty et al., 2001). Behavioral disturbances among residents are associated with high stress levels in staff (Brodaty et al., 2003) which, in turn, contribute to annual staff turnover rates of $20 \%$ for nurses and $25 \%$ for nursing assistants (Richardson and Martin, 2004). Since behavioral or psychological symptoms of dementia are associated with the person's morbidity and illbeing, staff burnout and turnover, and increased health care costs, finding alternative and effective methods that have the potential for reducing these behaviors and increasing well-being is warranted (Ayalon et al., 2006).

The use and experience of humor has been associated with a range of improvements in wellbeing including easing of depression, anxiety, loneliness, hopelessness, stress, and increased selfesteem and sense of control (Berk, 2001; Bennett et al., 2003). Older persons who have a better sense of humor and use humor as a coping mechanism are more likely to live longer, age well, and be more satisfied with their physical health and life in general (Celso et al., 2003). Humor therapy, a deliberate application of humorous stimulation, has been defined as "any intervention that promotes health and wellness by stimulating a playful discovery, expression or appreciation of the absurdity or incongruity of life's situations [which] may enhance health or be used as a complementary treatment of illness to facilitate healing or coping, whether physical, emotional, cognitive, social or spiritual" (Association for Applied and Therapeutic Humor, 2000). It is suggested that humor therapy requires the active involvement of the participant in humor creation, whereas external modalities (e.g. video comedy) are more passive and better defined as laughter therapy (Peacock, 2009). In this way, humor therapy differs from activities that encourage laughter without cognitive processing of humor, such as laughter yoga (Shahidi et al., 2011).

Empirical evidence for the positive effects of humor therapy in adults is scarce (Martin, 2004). While older adults may have poorer comprehension of humor than younger persons due to age-related cognitive decline, they have similar levels of enjoyment when finding something humorous (Shammi and Stuss, 2003) and the ability to generate humor persists through advanced stages of dementia (Hawkins and Graff-Radford, 2007). Engaging with humor offers older people, including those living with dementia in RACFs, an opportunity to meet a number of needs: activity, communication, pleasure, and social engagement. The use of humor and playfulness by nursing staff are important motivators in helping residents with cognitive impairment participate in functional activities (Galik et al., 2009). Interviews with nursing home residents indicate that residents seldom experience humor (Isola and Astedt-Kurki, 1997). This may be because RACFs offer few opportunities to experience humor and/or because residents do not always consider mainstream humor funny (e.g. television sitcoms).

Few studies have evaluated the efficacy of humor or laughter therapy in institutionalized older persons. Four studies (Boyd and McGuire, 1996; Houston et al., 1998; Ronnberg, 1998; Thomson, 2005), which used small sample sizes and relatively low quality methodology, showed some benefit - and only two of these studies allowed for active resident participation (Houston et al., 1998; Thomson, 2005). In a small study $(n=21)$ of a four-session clown visit program, there was an overall increase in positive behaviors and decrease in negative behaviors during the sessions for persons with severe dementia (Thomson, 2005). A controlled study $(n=61)$ found that five sessions of comical singing and dancing were associated with decreased self-rated anxiety and depression in residents (Houston et al., 1998). Residents $(n=87)$ who watched humorous movies three times a week for 12 weeks showed decreased negative affect over time compared to those who watched serious movies and controls who received usual care (Boyd and McGuire, 1996). Residents $(n=27)$ who watched recordings of humorous story-telling weekly for 12 weeks reported improved quality of life compared to those who watched conventional television or usual care (Ronnberg, 1998). Eight weekly sessions involving telling jokes and funny stories and discussion on prioritizing humor in daily life decreased reports of pain and perceived loneliness, and increased reported happiness and life satisfaction in residents in the intervention group $(n=36)$ compared to controls $(n=34)$ (Tse et al., 2010). None of the studies provided long-term follow-up data after cessation of the intervention.

Since humor therapy shows promise in improving well-being in older age and in dementia, the SMILE study rigorously evaluates its impacts in the RACF setting. The aims of the SMILE study are to evaluate the effect of humor therapy on:

1. Resident depression, quality of life, agitation, behavioral disturbance, and social engagement.

2. Staff mood, absenteeism, and turnover.

\section{Methods}

SMILE is registered with the Australian New Zealand Clinical Trials Registry (ACTRN12611000462987). 


\section{Study design}

A single-blinded two-group cluster-randomized design will be used. Data will be collected at three time points: baseline (week 0), post (week 13), and follow-up (week 26). The intervention cannot be isolated to specific residents within facilities, so treatment allocation will be applied at the facility level.

\section{Ethical considerations}

The protocol has been approved by the human research ethics committee of the University of New South Wales, Sydney, Australia, and will be reviewed by governance at each participating site including the resident committee if appropriate. Written consent will be obtained for each participating resident; if classified as a vulnerable person (e.g. dementia diagnosis), a responsible person will provide written consent, and the resident's assent will be obtained directly (e.g. nod) and/or indirectly (e.g. continuing willingness to participate) prior to any humor therapy session or assessment occasion. If a resident refuses on three consecutive occasions then the resident's ongoing participation will be reviewed.

\section{Setting and recruitment of RACF}

The study will be conducted in government accredited RACFs throughout metropolitan Sydney, NSW, Australia. A candidate list will be compiled from publicly accessible aged-care listings (DPS Publishing, 2010) and supplemented by the researchers' professional networks. Of these, facilities meeting the following screening criteria will be mailed an invitation to register interest to participate:

1. government-accredited residential care;

2. located within one-hour's drive from the researchers' university;

3. not known to be enrolled in another intervention study (to reduce risk of contaminating study outcomes).

Sites that registered interest will be further screened for eligibility. Facilities are to be excluded if they:

1. are enrolled in another intervention study;

2. cater specifically for a particular ethnic and cultural minority group;

3. cater specifically for particular medical conditions except for dementia (e.g. Huntington's disease);

4. are scheduled to undergo a management or cultural change in the study timeframe;

5. cannot secure site-specific governance permission; or

6. will not agree to study terms.

\section{Recruitment of residents}

The intervention has been designed to be delivered to up to 20 residents living together within an RACF unit. From each participating RACF a defined geographical area (ward, corridor or floor) will be selected as their "SMILE neighborhood," based on discussion with managers and staff. SMILE neighborhoods will meet the following criteria:

1. accommodating fewer than 40 permanent residents;

2. permit interaction between residents and staff nominated for LaughterBoss training;

3. not foreshadowed for change (e.g. in management or care plans);

4. not scheduled for major structural alterations (e.g. capacity expansion).

Residents will be excluded if they are:

1. younger than 50 years of age;

2. admitted to full-time care less than 12 weeks prior (with respite care before permanent admission counting toward the 12 weeks);

3. exhibiting behavior presenting a risk to study personnel (e.g. unpredictable aggression);

4. foreshadowed to move out of SMILE neighborhood within six months;

5. experiencing severe communication obstacles, e.g. bed-bound vegetative state or severely impaired in at least two out of three of impairments for sight, hearing, and speech;

6. acutely unwell, including frequent hospitalization and terminal illness care plan;

7. floridly symptomatic psychiatrically, or a known fear of clowns or strangers;

8. under public guardianship with no responsible person; or

9. non-English speaking with no alternative communication (e.g. pointing) or bilingual informant.

\section{Recruitment of RACF staff}

Up to ten staff with a primary care role working in the SMILE neighborhood will be invited to participate by completing a short anonymous survey at each assessment occasion. A staff contact will be asked to distribute the surveys and reply paid envelopes and keep a list of the names of participating staff matched to identifier numbers on each survey.

\section{Humor therapy intervention}

The intervention comprises two key components: LaughterBoss training for one RACF staff member per facility and weekly ElderClown humor therapy sessions. The LaughterBoss training program was developed and delivered by PS (Spitzer, 2011). The LaughterBoss will be trained and enabled to have fun with residents, shown techniques for enhancing communication and positive diversion, 
shown techniques and tricks for incorporating humor and play into facility routines (e.g. during medication rounds, or holding funny hat dress-up days), and encouraged to experiment with ways to engage playfully with residents. LaughterBosses will be informally mentored by ElderClowns during their visits and encouraged to continue play themes between and after visits.

Staff members will be nominated for LaughterBoss training by RACF managers after discussion with study staff, with encouragement to nominate a person who:

1. is keen to volunteer for such a role;

2. spends significant time in the SMILE neighborhood;

3. enjoys interacting with residents;

4. is available to attend a one-day training seminar on humor therapy;

5. may have protected time from duties to participate in humor therapy sessions.

The study will pay for all LaughterBoss costs including backfill of their positions so that LaughterBosses may participate in the humor therapy sessions.

Experienced Clown Doctors will be selected to work as ElderClowns in the study, and given additional training for providing humor therapy in residential aged care. They will be mentored by JPB.

ElderClowns will conduct weekly two-hour sessions over 12 weeks, engaging residents and staff in humor via music, mime, props, and other techniques. ElderClown sessions will vary depending upon the residents, facility routine, and geographical layout of the facility; however, the sessions typically will have the following pattern: the ElderClown accompanied by the LaughterBoss will begin by engaging with the group of residents present in common areas. Interactions may involve conversations and jokes and songs. The ElderClown will then target interactions with individual residents, first focusing on those in the common areas, then visiting residents in their rooms. The length of the interactions will depend upon the level of engagement and capacity for concentration of the resident and the number of residents to be visited at that facility. Some typical ways of interacting with residents would be using popular old songs, physical games such as balloon tennis, and use of puppets, hats, and other props.

ElderClowns are skilled improvisers and will tailor their interventions according to the abilities and interests of the residents. Information about each resident will be obtained from the resident file and through LaughterBoss briefings before and debriefings after each session. ElderClowns will prepare strategies to engage individual residents - for instance, preparing a conversation about their favorite dog breed, song from their culture, or bringing in an item relevant to their previous occupation such as tools. Strategies will be reviewed and amended after each visit and documented in LaughterCharts.

\section{Control group}

Control group RACFs will provide usual care to residents and will be asked not to engage residents in humor therapy. Staff will be offered LaughterBoss training once follow-up assessments are completed for that RACF.

\section{Randomized allocation to intervention}

The 36 SMILE neighborhoods will be stratified by size ( $<18$ or $>18$ beds) and care type (high-care only and mixed high- and low-care; or low-care only), assigned an RACF number and randomized. The randomization will be conducted by LFL with the deidentified RACF numbers using computergenerated random numbers.

Within the study team, treatment allocation will be known only to the principal investigator (HB), the researcher performing the randomization (LFL), and to the administrative support person. Research staff conducting onsite assessments (BG, ANC) will be blind to treatment group allocation and RACFs will be given ongoing reminders to maintain the blinding.

\section{Assessment}

Outcome measures

Outcome measures will be collected at the three time points, baseline (week 0), post (week 13), and follow-up (week 26), and comprise standardized instruments selected for demonstrated validity and reliability for persons with dementia living in residential aged care. Psychologists trained and supervised by experienced clinicians including psychologists, nurses, and a psychogeriatrician will collect data.

Information will be collected through interviews with the resident (if able and willing) and a reliable staff informant, direct observation, and from resident clinical files. Whenever possible, the same staff informant will be interviewed at each time point by the same researcher.

Depression: The 19-item Cornell Scale for Depression in Dementia (CSDD) is a clinician-rated tool specifically developed to assess signs and symptoms of major depression in persons with dementia based on all available evidence (Alexopolous et al., 1988).

Agitation: The 29-item Cohen-Mansfield Agitation Inventory (CMAI) (Cohen-Mansfield, 1989) is an 
informant-rated tool on the frequency of agitated behaviors.

Social engagement: In the absence of a validated measure for social engagement in residential aged care settings, the eight-item withdrawal subscale of the Multidimensional Observation Scale for Elderly Subjects (MOSES) will be used (Helmes et al., 1987). The items address preference for solitude, initiative in social interaction, and interest in events.

Quality of life: Proxy- (30-item) and self-report (29item) versions of the Dementia Quality of Life scale (DEMQOL) will be used to measure quality of life (Banerjee et al., 2006).

Psychopathology: The Neuropsychiatric Inventory Nursing Home (NPI-NH) (Cummings et al., 1994) 12-item version will be used to measure informant ratings of the severity and frequency of behavioral disturbances.

ADDITIONAL RESIDENT MEASURES

Additional resident measures will also be completed at all three time points and comprise: measures of disease comorbidity, medications, impairment, and general demographics (baseline only). Demographic data collection will include reviewing the most recent RACF-completed Aged Care Funding Instrument (ACFI) assessment, which is used to determine public funding amounts for residential aged care places. The ACFI contains two measures that are also part of the SMILE assessment battery: the CSDD (Alexopolous et al., 1988) and the cognitive subscale of the Psychogeriatric Assessment Scale (PAS) (Jorm et al., 1997).

Comorbidity: The following information will be extracted from patient charts: diagnosis of dementia and other neurodegenerative disorders (e.g. Parkinson's disease), psychiatric diagnoses (e.g. depression, anxiety, psychosis), illness comorbidity (e.g. diabetes, cardiovascular disease), and history of substance use (e.g. tobacco, alcohol).

Medications: Information about medications generally and psychotropics in particular will be extracted from medication charts, including regimen (regular versus pro re nata), dose, and frequency in the two weeks prior to the chart audit.

Impairment (cognitive, functional, and communicative): Cognitive impairment will be assessed by researchers via direct resident interview using the 21-item cognitive subscale of the PAS (Jorm et al., 1997). Higher scores indicate higher levels of cognitive impairment, with four score-based category rankings as follows: 0 $3=$ no/minimal impairment, $4-9=$ mild impair- ment, $10-15=$ moderate impairment, and 16$21=$ severe impairment. Residents incapable of engaging with the PAS will be ranked as severely impaired. For residents who have completed the PAS via an ACFI assessment within three months prior to SMILE baseline, the ACFI score will be used.

Functional impairment will be assessed using the ten-item Barthel Index (Mahoney and Barthel, 1965), measuring activities of daily living (ADL) and mobility. Additionally, we have developed three items to assess sight, hearing, and speech with Barthel-style scoring for speech (ease of understanding resident), sight (level of difficulty with prescribed correctional device), and hearing (level of difficulty with prescribed aids).

Disease severity: The Global Deterioration Scale (GDS) (Reisberg et al., 1982) will be used to classify residents on relative severity of dementiarelated cognitive impairment and concomitant-care needs. The GDS is a seven-stage rating scale based on a composite of clinical judgment and formal assessment, where stages 1-4 are "predementia" and stages 5-7 represent a time when "a person can no longer survive without assistance." The GDS staging will be based on reviewing scores on other measures for cognitive and functional impairment, observation, and the resident's most recent ACFI assessment.

BEHAVIORAL OBSERVATION OF RESIDENTS A computer-assisted behavioral observational tool named the Behavior, Engagement, and Affect Measure (BEAM) has been developed specifically for this study. The BEAM will be used to collect 30 minutes of behavioral observations on each resident at the three time points. The development and validation of this tool will be described in a separate paper.

HUMOR THERAPY INTERVENTION RELATED MEASURES

LaughterCharts: In the intervention RACFs, LaughterBosses and ElderClowns will complete purposespecific LaughterCharts for every resident after each ElderClown humor therapy session. The LaughterCharts will record session content (e.g. music, conversation), format (individual or group), a resident reactions checklist (e.g. smiled, confused), and two global ten-point ratings of intervention success $(1=$ extremely unsuccessful; $10=$ extremely successful) rated separately by the ElderClown and LaughterBoss. Qualitative descriptive field notes of the session and ideas for future humor interactions will be recorded. 
FACILITY AND STAFF-RELATED MEASURES Staff survey: A six-item purpose-specific anonymous staff survey has been developed to estimate staff mood and RACF atmosphere, using level of agreement on a five-point Likert scale from "strongly agree" to "strongly disagree." The items are: "I felt happy at work," "I enjoyed interacting with the residents," "I found it challenging to look after the residents," "I felt enthusiastic about my job," "My workplace felt stressful," "My workplace had a positive atmosphere."

Staff morale, staff leave, and staff turnover: It will not be feasible to extract SMILE neighborhood level data on staff leave and turnover from the records of most RACFs. Hence global unit-level ratings of staff morale, turnover, and leave will be obtained from the RACF manager and/or LaughterBoss for three time periods: prebaseline, baseline to post, and post to follow-up. This will be rated on a five-point scale (very low-very high) to estimate change.

Staff mix: Information will be collected about staffing (number of positions and duties) within the SMILE neighborhood for morning, afternoon, and night rosters.

RACF physical environment assessment: The 72item Environmental Audit Tool (EAT) (Fleming, 2011) will be used by researchers to rate RACF environment on ten ideal design principles for housing confused and disturbed elderly persons.

Humor therapy intervention implementation: Researchers have developed a 29-item checklist to document humor therapy implementation processes in intervention RACFs. The items address a range of RACF engagement and compliance behaviors, ranging from managers giving the LaughterBoss protected time to accompany the ElderClown on humor therapy sessions to the level of LaughterBoss-initiated humor therapy. Each item was rated on a three-point scale using best available information.

\section{Statistical analysis}

SAMPLE SIZE

A sample size of 36 RACF units with nine to ten residents in each has been estimated to provide $80 \%-99 \%$ power to detect a medium effectsize (Cohen's $\mathrm{d}=0.5$ ) difference on continuous outcomes with significance level set at 0.05 taking into account the clustering of residents within facilities (Hayes and Bennett, 1999), and based on variance between units on the outcome measures obtained from a previous multisite RACF study (Chenoweth et al., 2009).

\section{OUTCOME ANALYSES}

An intention-to treat approach will be used to test for main effects on key outcome measures, so all residents/staff with baseline data will be included in the analyses. Multilevel modeling will be conducted using SPSS and SASS to test the interaction between treatment and time, accounting for clustering of assessment points within residents and residents within facilities (Murray, 1998). Multilevel modeling allows for residents with missing data at each time point to be included in the overall analysis. Patterns of missing data will be examined to ascertain potential bias of results. Potential fixed and time-dependent covariates will be tested for and adjusted for in the analysis.

\section{Discussion}

SMILE is the world's largest rigorous study of humor therapy in any setting. The humor intervention combines the input of an external experienced performer delivered in partnership with a trained staff member who sustains the humor therapy between sessions and after the formal intervention is completed.

SMILE measures resident outcomes using industry standard scales, supplemented by observational data collected using a new computerized tool and qualitative interviews. It also examines staff outcomes in relation to the humor therapy intervention. In addition, the study will examine what resident characteristics contribute to greater engagement during the humor therapy sessions; whether engagement during humor therapy sessions are associated with greater improvement on outcomes, and what aspects of management and staff behavior at a facility level contribute to successful outcomes.

The SMILE study will provide evidence as to whether humor therapy is a worthwhile psychosocial intervention in the delivery of quality residential aged care.

\section{Conflict of interest}

Peter Spitzer and Jean-Paul Bell were both employed by the not-for-profit organization the Humour Foundation and received payments for delivery of humor therapy services. Peter Spitzer is a consultant and board member of the Humour Foundation. Jean-Paul Bell and Lee-Fay Low are board members of the not-for-profit Arts Health Institute that also provides humor therapy services. 


\section{Description of authors' roles}

Henry Brodaty and Peter Spitzer conceptualized the study and Lee-Fay Low and Henry Brodaty designed the study. Henry Brodaty, Lee-Fay Low, Lynn Chenoweth, Richard Fleming, and Peter Spitzer contributed to study design; Henry Brodaty, Lee-Fay Low, Lynn Chenoweth, Richard Fleming, and Peter Spitzer secured funding. Belinda Goodenough and Lee-Fay Low developed the study protocol. Belinda Goodenough managed and Anne-Nicole Casey contributed to study implementation. Peter Spitzer and Jean-Paul Bell developed and delivered humor therapy services. Belinda Goodenough and Lee-Fay Low drafted this paper and all authors contributed, read, and approved the final version of the paper.

\section{Acknowledgments}

This study was funded by the National Health and Medical Research Council ID 568787. The authors thank Linda Nattrass, Mark Do, Fiona White, and Lindy Bergman for assistance with recruitment and data collection; Nick Witt, Michelle Witt, and Laurie Kilfoyle and the Humour Foundation for contributing to humor therapy sessions; Jiska Cohen-Mansfield, Adrienne Withall, and Kim Burns for input on measure selection; and Madeleine King for her advice on statistical analysis. The authors are grateful to the families, residents, and staff of RACFs who took part.

\section{References}

Alexopolous, G., Abrams, R., Young, R. and Shamoian, C. (1988). Scale for depression in dementia. Biological Psychiatry, 23, 271-284.

Association for Applied and Therapeutic Humor (2000). Association for Applied and Therapeutic Humor. Available at: http://www.aath.org/general-information; last accessed 20 April, 2012.

Ayalon, L., Gum, A. M., Feliciano, L. and Areán, P. A. (2006). Effectiveness of nonpharmacological interventions for the management of neuropsychiatric symptoms in patients with dementia: a systematic review. Archives of Internal Medicine, 166, 2182-2188.

Banerjee, S. et al. (2006). Quality of life in dementia: more than just cognition. An analysis of associations with quality of life in dementia. Fournal of Neurology, Neurosurgery and Psychiatry, 77, 146-148.

Bennett, M., Zeller, J., Rosenberg, L. and McCAnn, J. (2003). The effect of mirthful laughter on stress and natural killer cell activity. Alternative Therapies in Health and Medicine, 9, 38-44.

Berk, R. A. (2001). The active ingredients in humor: psychophysiological benefits and risks for older adults. Educational Gerontology, 27, 323-339.
Boyd, R. and McGuire, F. (1996). The efficacy of humor in improving psychological well-being of residents of long-term care facilities. Fournal of Leisurability, 23, 1-15.

Braithwaite, J. (2001). Regulating nursing homes: the challenge of regulating care for older people in Australia. BMF, 323, 443-446.

Brodaty, H., Draper, B. and Low, L. F. (2003). Nursing home staff attitudes towards residents with dementia: strain and satisfaction with work. Fournal of Advanced Nursing, 44, 583-590.

Brodaty, H. et al. (2001). Psychosis, depression and behavioural disturbances in Sydney nursing home residents: prevalence and predictors. International fournal of Geriatric Psychiatry, 16, 504-512.

Celso, B. G., Ebener, D. J. and Burkhead, E. J. (2003). Humor, coping, health status, and life satisfaction among older adults residing in assisted living facilities. Aging and Mental Health, 7, 438-445.

Chenoweth, L. et al. (2009). Caring for Aged Dementia Care Resident Study (CADRES) of person-centred care, dementia-care mapping, and usual care in dementia: a cluster-randomised trial. The Lancet Neurology, 8, 317-325.

Cohen-Mansfield, J. (1989). A description of agitation in a nursing home. Fournal of Gerontology, 44, M77-M84.

Cullen, D. (2007). The financial impact of entering aged care. Australasian fournal on Ageing, 26, 145-147.

Cummings, J. L., Mega, M., Gray, K., Rosenberg-Thompson, S., Carusi, D. A. and Gornbein, J. (1994). The neuropsychiatric inventory: comprehensive assessment of psychopathology in dementia. Neurology, 44, 2308-2314.

DPS Publishing (2010). DPS Guide to Aged Care: NSW $\mathcal{E}$ $A C T$. Adelaide, Australia: DPS Publishing.

Fleming, R. (2011). An environmental audit tool suitable for use in homelike facilities for people with dementia. Australasian fournal on Ageing, 30, 108-112.

Galik, E. M., Resnick, B. and Pretzer-Aboff, I. (2009). 'Knowing what makes them tick': motivating cognitively impaired older adults to participate in restorative care. International Fournal of Nursing Practice, 15, 48-55.

Hawkins, D. B. and Graff-Radford, N. R. (2007). The ability to pun may be retained in Alzheimer disease. Neurocase, 13, 50-54.

Hayes, R. J. and Bennett, S. (1999). Simple sample size calculation for cluster-randomized trials. International Fournal of Epidemiology, 28, 319-326.

Helmes, E., Csapo, K. and Short, J. A. (1987). Standardization and validation of the Multidimensional Observation Scale for Elderly Subjects (MOSES). Fournal of Gerontology, 42, 395-405.

Houston, D. M., McKee, K. J., Carroll, L. and Marsh, H. (1998). Using humour to promote psychological wellbeing in residential homes for older people. Aging and Mental Health, 2, 328-332.

Isola, A. and Astedt-Kurki, P. (1997). Humour as experienced by patients and nurses in aged nursing in Finland. International fournal of Nursing Practice, 3, 29-33.

Jorm, A. F., Mackinnon, A. J., Christensen, H., Henderson, A. S., Jacomb, P. A. and Korten, A. (1997). The Psychogeriatric Assessment Scales (PAS): further data on psychometric properties and validity. International fournal of Geriatric Psychiatry, 12, 93-100. 
Mahoney, F. I. and Barthel, D. W. (1965). Functional evaluation: the Barthel Index. Maryland State Medical fournal, 14, 61-65.

Martin, R. (2004). Sense of humor and physical health: theoretical issues, recent findings, and future directions. Humor, 17, 1-19.

Murray, D. M. (1998). Design and Analysis of Group-Randomised Trials. Oxford: Oxford University Press.

Peacock, L. (2009). Serious Play. Chicago: Intellect.

Reisberg, B., Ferris, S. H., de Leon, M. J. and Crook, T. (1982). The Global Deterioration Scale for assessment of primary degenerative dementia. American fournal of Psychiatry, 139, 1136-1139.

Richardson, S. and Martin, B. (2004). The Care of Older Australians: A Picture of the Residential Aged Care Workforce. Adelaide, Australia: The National Institute of Labour Studies.

Ronnberg, L. (1998). Quality of life in nursing-home residents: an intervention study of the effect of mental stimulation through an audiovisual programme. Age and Ageing, 27, 393-397.
Shahidi, M. et al. (2011). Laughter yoga versus group exercise program in elderly depressed women: a randomized controlled trial. International fournal of Geriatric Psychiatry, 26, 322-327.

Shammi, P. and Stuss, D. (2003). The effects of normal aging on humor appreciation. Fournal of the International Neuropsychological Society, 9, 855-863.

Snowdon, J. and Fleming, R. (2008). Recognising depression in residential facilities: an Australian challenge. International fournal of Geriatric Psychiatry, 23, 295-600.

Spitzer, P. (2011). The laughterboss. In T. Adams and H. Lee (eds.), Creative Approaches in Dementia Care (pp. 32-53) New York: Palgrave MacMillan.

Thomson, R. (2005). Evaluation of the use of a clown therapy group with dementia sufferers. NHS Borders Psychological Services, 3-4.

Tse, M. Y., Lo, A. P. K., Cheng, T. L. Y., Chan, E. K. K., Chan, A. H. Y. and Chung, H. S. W. (2010). Humor therapy: relieving chronic pain and enhancing happiness for older adults. Fournal of Ageing Research, doi: $10.4061 / 2010 / 343574$. 